\title{
Histología de branquias, hígado y riñón de juveniles del pez neotropical Colossoma macropomum (Characiformes, Characidae) expuesto a tres temperaturas
}

\author{
Luz-Marina Rojas*1, Claunis Mata ${ }^{2}$, Aridays Oliveros ${ }^{3} \&$ Raquel Salazar-Lugo ${ }^{2,3}$ \\ 1. Laboratorio de Histología, Instituto de Investigaciones en Biomedicina y Ciencias Aplicadas "Dra. Susan Tai", \\ Universidad de Oriente; Imarinarojas@yahoo.com \\ 2. Departamento de Bioanálisis, Núcleo de Sucre Universidad de Oriente. Laboratorio de proteínas e Inmunotoxicidad; \\ claunismata@hotmail.com \\ 3. Postgrado de Biología Aplicada, Universidad de Oriente; rsalazarlugo50@gmail.com, arydaises@yahoo.com \\ * Correspondencia
}

Recibido 18-I-2012. Corregido 20-IX-2012. Aceptado 24-X-2012.

\begin{abstract}
Histology of gill, liver and kidney in juvenile fish Colossoma macropomum exposed to three temperatures. Water temperature is an important factor that affects growth and antioxidant enzyme activities in fish, and when adverse, it may trigger diseases in fish populations. C. macropomum is a freshwater neotropical fish widely distributed in South America and abundant in river basins as the Amazon and Orinoco. It is highly used for intensive aquaculture development and is a very important product for the local riverside economy in Venezuela. The purpose of our study was to examine the water temperature effect on gills, liver and kidneys of juvenile fishes of C. macropomum. Eighteen juveniles with biometrical index of $17.87 \pm 7.88 \mathrm{~cm}$ and $87.69 \pm 34.23 \mathrm{~g}$ were respectively exposed to three culture temperatures $\left(\mathrm{T} 18, \mathrm{~T} 29\right.$ and $\left.\mathrm{T} 35^{\circ} \mathrm{C}\right)$ during a period of 21 days. Histological analyses on gills, liver and kidney were made according to standard methodologies. Our results showed that these tissues exhibited normal citoarchitecture at T29. On the contrary, T18-gills displayed brachiallipid droplets inside brachial epithelium; and disorganization in the brachial tissue was observed at T35. Furthermore, we observed two kinds of hepatocytes (dark and light) on $\mathrm{T} 18^{\circ} \mathrm{C}$-liver. The T35-liver samples showed cytoplasmatic granulation and damages in cytoplasmatic membrane. Kidney samples from T18 observed alterations in the cellular distribution of the hematopoietic tissue; while, at T35, the most important feature observed was the disorganization of the glomerular structure. We concluded that T18 and T35 are respectively critical and severe temperatures to C. macropomum; besides, the most sensible tissues to changes induced by temperature in this species were the liver and gills. Rev. Biol. Trop. 61 (2): 797-806. Epub 2013 June 01.
\end{abstract}

Key words: Colossoma macropomum, histology, temperature, liver, kidney, gill.

Los peces, por ser organismos poiquilotermos, son altamente influidos por los cambios en la temperatura, pudiendo este factor ser determinante en el predominio del sexo en algunas especies, porque puede afectar la producción de estrógenos (Azaza et al. 2008, Körner et al. 2008, Rougeot et al. 2008). Igualmente, los cambios de temperatura por encima o por debajo de los valores adecuados para el desarrollo de la especie, pueden inducir cambios en la respuesta inmunológica de los peces; estos cambios pueden ser una respuesta inmunosupresora lo cual facilitaría el desarrollo de enfermedades oportunistas (Marcogliese 2008, Pérez-Casanova et al. 2008) o una respuesta estimuladora del sistema inmune y de esta manera el organismo puede protegerse de ciertos virus (Sano et al. 2009).

El pez Colossoma macropomum, conocido en Venezuela con el nombre de cachama y en otros países latinoamericanos con el nombre de tambaqui, es un pez migratorio que se desplaza 
aguas arriba en épocas de verano procurando mejores condiciones para su sobrevivencia, a la vez que se prepara para su reproducción, que se cumple cíclicamente cada año en la temporada de invierno. Esta especie está ampliamente distribuida desde el Orinoco por toda la cuenca amazónica, y ha representado, durante muchos años, un excelente y apetecido producto de la pesca fluvial, principalmente en los ríos Guanare, Portuguesa, Apure, Orinoco y sus afluentes, ofertándose con apreciable abundancia en los mercados locales y algunas ciudades de importancia en el país (Fernández 1986). Este es uno de los peces tropicales de agua dulce con mayor éxito en el cultivo en aguas continentales tropicales (Tafur-González et al. 2009, Andrade de Pasquier et al. 2011).

El análisis histológico de órganos dianas (aquel que reacciona a un estímulo específico bien sea externo o interno) constituye una herramienta sensible y específica mediante la cual se pueden apreciar alteraciones en los tejidos. Los órganos que reaccionan a ese tóxico son órganos diana. Algunos órganos, más que otros, también podrían ser muy susceptibles a cambios inducidos por factores abióticos como la temperatura. Entre los órganos a considerar para las evaluaciones histológicas, tanto en vertebrados como en invertebrados acuáticos, están las branquias, el hígado y el riñón: las branquias debido a que son los órganos directamente expuestos y los más susceptibles a las variaciones ambientales (Cajaraville et al. 2000); el hígado, dada a la importancia que tiene en los procesos de desintoxicación, y los riñones, porque además de su función fisiológica de excreción, también actúa como órgano hematopoyético de los peces (Goessling \& North 2011).

C. macropomum es un pez de comportamiento migratorio, puede vivir en diferentes ambientes (incluyendo contaminados), soporta un amplio rango de $\mathrm{pH}$ y baja concentración de oxígeno (Useche 2000). Sin embargo, se tiene poca información acerca de la susceptibilidad de esta especie a los cambios de temperatura, tanto en la naturaleza como bajo medio ambiente controlado. En vista del gran potencial económico que tiene C. macropomum, el objetivo del presente estudio es determinar cuál de los tres órganos diana evaluados; branquias, hígado y riñón, es el más sensible a los efectos de la temperatura bajo ambiente controlado.

\section{MATERIALES Y MÉTODOS}

Obtención y mantenimiento de los peces: Se utilizaron 36 ejemplares juveniles de C. macropomum (Cuvier, 1818), con índices biométricos de $17.87 \pm 7.88 \mathrm{~cm}$ y $87.69 \pm 34.23 \mathrm{~g}$, los cuales fueron suministrados por el Instituto Nacional de Investigaciones Agrícolas (INIA), estación experimental Delta Amacuro, Tucupita, estado Delta Amacuro. Los peces se trasladaron en bolsas con agua aireada hasta el Laboratorio de Camarones Dulceacuícolas (LCD), situado en el Núcleo de Sucre de la Universidad de Oriente.

Manejo y aclimatación: En el laboratorio, las bolsas con los peces fueron colocadas en tanques de $1000 \mathrm{~L}$ de capacidad, preparados con agua declorada y aireada; transcurrido el tiempo de 30min., los organismos fueron sacados de las bolsas y colocados directamente en el tanque.

Al agua del tanque se le agregó Azitromicina (1g) y cloranfenicol (1g) a fin de prevenir infecciones bacterianas y fúngicas. Durante el tiempo de aclimatación se realizaron recambios del agua entre un $75-80 \%$ del volumen total. Los especímenes fueron alimentados dos veces al día con alimento granulado (cachamarina, marca Purina) el cual fue suministrado considerando el $40 \%$ de su peso corporal; los peces se sometieron a un fotoperíodo de 12/12 horas y a una temperatura de $29^{\circ} \mathrm{C}$. Al finalizar el tiempo de aclimatación, los peces fueron considerados aptos para los bioensayos.

Bioensayo: Individuos de tallas similares de C. macropomum (36 peces) fueron expuestos durante 21 días a tres temperaturas, $18^{\circ} \mathrm{C}$ (T18), $29^{\circ} \mathrm{C}$ (T29) y $35^{\circ} \mathrm{C}(\mathrm{T} 35)$ en acuarios de $50 \mathrm{~L}$ de agua (base: $50 \times 24 \mathrm{~cm}$; altura $28 \mathrm{~cm}$ ) según Salazar-Lugo et al. (2009). T18 se logró 
en acuarios dotados con un motor adaptado para enfriamiento y T35 utilizando calentadores para acuarios marca Elite. Se colocaron dos acuarios por cada temperatura cada uno conteniendo seis peces. Los peces fueron aclimatados gradualmente a las temperaturas de $18^{\circ} \mathrm{C}$ y $35^{\circ} \mathrm{C}$. El pH del agua se mantuvo en 7.7 y la dureza fue de 106ppm.

Análisis histológicos: Los análisis histológicos fueron realizados de acuerdo a técnicas convencionales para la microscopía electrónica previamente utilizadas (Rojas et al. 1997; Bravo et al. 2005). Inmediatamente después del sacrificio (por decapitación), se procedió a tomar las muestras de branquias, hígado y riñón. El procedimiento completo se realizó a una temperatura de $21^{\circ} \mathrm{C}$. Cada muestra fue cortada en secciones de $2 \mathrm{~mm}^{2}$ aproximadamente, las cuales se colocaron en glutaraldehído al $3 \%$ a un $\mathrm{pH} 8.02$ por $2 \mathrm{~h}$. Luego, fueron lavadas con amortiguador fosfato de concentración de $0.1 \mathrm{~mol} / \mathrm{L}$ y $\mathrm{pH} 7.4$ durante $15 \mathrm{~min}$, y post-fijadas durante $1 \mathrm{~h}$ en tetraóxido de osmio $\left(\mathrm{OsO}_{4}\right)$ al $1 \%$. Posteriormente, se lavaron en amortiguador y se deshidrataron con una batería de etanoles a concentraciones crecientes desde 50 hasta $100 \%$, durante $5 \mathrm{~min}$ cada uno. Para la infiltración, las muestras se colocaron en óxido de propileno por $15 \mathrm{~min}$, luego se pusieron en una mezcla de 1:1 resina epóxica-óxido de propileno por $2 \mathrm{~h}$, y luego en resina epóxica pura, durante 1h (Luft 1961). Finalmente, la inclusión se hizo colocando las muestras en moldes de silicona con resina epóxica y la polimerización se realizó en una estufa a $60^{\circ} \mathrm{C}$ por $48 \mathrm{~h}$. Se practicaron cortes semifinos $(0.7 \mu \mathrm{m})$ con cuchilla de vidrio en un ultra micrótomo Leica UM-UC6. Los cortes se tiñeron con azul de toluidina (1\%), y fueron observados con un fotomicroscopio modelo Axioskop FL40 (Carl Zeiss, Germany), fotografiados con una cámara Cannon y procesados por medio de un software analizador de imágenes Axio Vision LE Cannon Modul (Carl Zeiss, Germany).

\section{RESULTADOS}

Histología de branquias: Las branquias de T29 (considerada la temperatura normal)se observaron en buen estado mostrando células cartilaginosas (CC) rodeadas por tejido cartilaginoso (TC). Este tejido a su vez se encuentra bordeado por una fina capa de células epiteliales (CE) (Fig. 1-A). Por otro lado, se evidenciaron capilares (C) y eritrocitos (ER), ambos con morfología típica normal en los peces (Fig. 1-B).

En T18, a diferencia de T29, se observaron gotas lipídicas (GL) de tamaño considerable inmersas en el tejido branquial (TB); sin embargo, la estructura de TB parece no haberse afectado por esta temperatura (Figs. 1-C y 1-D).

En T35 se evidenciaron CC con borde irregular y que perdieron su forma típica redondeada (Fig. 1-E); además, se observaron ER con forma anormal comparados con T29, posiblemente dañados por efecto de la temperatura (Fig. 1-F).

Histología de hígado: El tejido hepático de los peces T29 mostró una apariencia normal, con una coloración uniforme. Los hepatocitos (H) presentaron un núcleo con cromatina laxa (eucromatina), redondeado, centrado y con la presencia de un nucléolo osmiofílico (Fig. 2).

En contraste con T29, el tejido hepático de T18 no presentó una coloración uniforme. Se observaron, hepatocitos claros y hepatocitos oscuros (Fig. 2). El aspecto claro-oscuro se evidenció a pesar de que todas las muestras de tejido hepático, expuestas a las diferentes temperaturas, fueron histológicamente tratadas del mismo modo y al mismo tiempo, descartándose así un error en el proceso de fijación del tejido. Se infiere, que la baja temperatura pudo haber influido en la bioquímica de los hepatocitos alterando, la coloración uniforme observada en T29 (Fig. 2). 

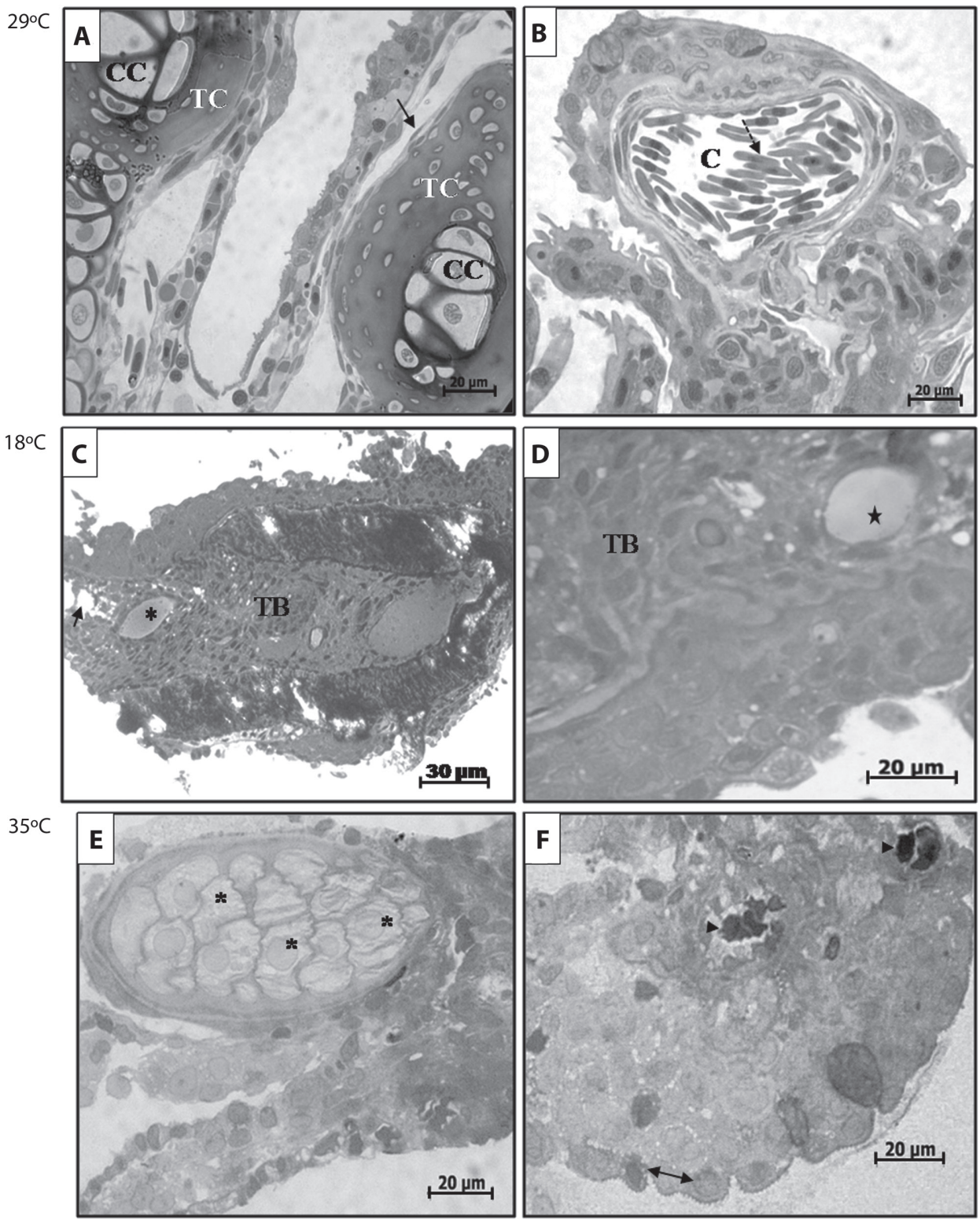

Fig. 1. Corte transversal de branquias de C. macropomum expuestos a diferentes temperaturas. 29 $^{\circ} \mathrm{C}$. A. Detalle de las células cartilaginosas (CC) en los filamentos branquiales; al tejido cartilaginoso (TC), epitelio branquial (flecha); B. Detalle de un capilar (C) en el extremo terminal de un filamento branquial. Obsérvese los eritrocitos con morfología normal. C. y D. $\mathbf{1 8}^{\circ} \mathrm{C}$. Detalles del filamento branquial donde se observan una gotas lipídicas (asterisco) inmersa en el tejido branquial (TB). E. y F. $\mathbf{3 5}^{\circ} \mathrm{C}$. Detalle del tejido branquial. E. Tejido cartilaginoso de un filamento branquial donde se evidencia la deformación de sus células (asterisco) y F. Se observa un capilar con eritrocitos de morfología anormal.

Fig. 1. Gills cross section of C. macropomum exposed to different temperatures. $2^{\circ} \mathrm{C}$. A. Details of cartilage cells $(\mathrm{CC})$ in the gill filaments, cartilage tissue (TC), gill epithelium (arrow); B. Detail of a capillary (C) at the terminal endofagillfilament. Note erythrocytes with normal morphology. C. and D. $\mathbf{1 8}^{\circ} \mathbf{C}$. Details of gill filaments how in glipid droplets (asterisk) embedded in the gill tissue (TB). E. and F. $3^{\circ} \mathrm{C}$. Detail of gill tissue. E. Cartilage tissue of a gill filament showing cell deformations (asterisk) and F. Capillary showing abnormal morphology erythrocytes. 

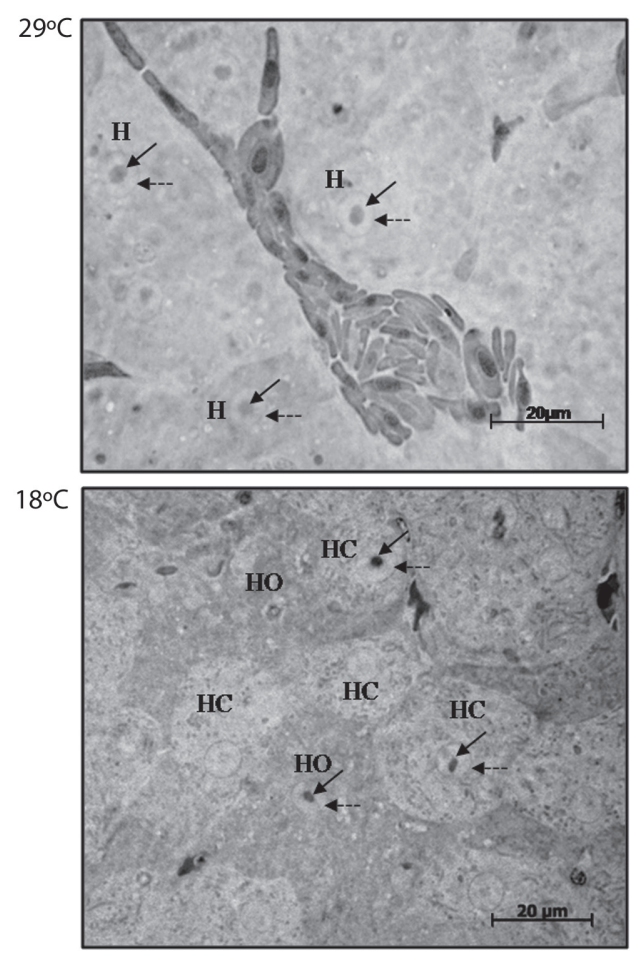

$35^{\circ} \mathrm{C}$

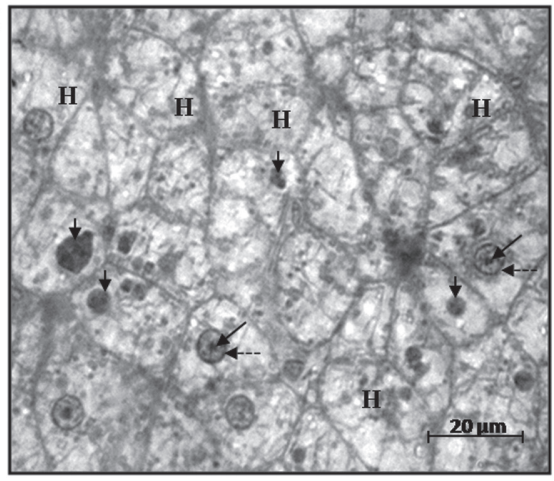

Fig. 2. Corte transversal de hígado de C. macropomum expuestos a diferentes temperaturas. $2^{\circ} \mathrm{C}$. Se observan hepatocitos $(\mathrm{H})$ con núcleos bien definidos (flecha punteada) conteniendo nucléolos (flechas continuas). $18^{\circ} \mathrm{C}$. Se evidencian hepatocitos claros (HC) y hepatocitos oscuros (HO). $\mathbf{3 5}^{\circ} \mathbf{C}$. Se observan hepatocitos con citoplasma claro posiblemente como producto de la pérdida de la integridad del citoplasma. El núcleo no muestra una apariencia uniforme (flechas continuas) y el nucléolo se nota difuso (flechas punteadas). Nótese la presencia de núcleos picnóticos (flechas cortas).
El grupo T35, presentó anomalías distintas a las observadas en T18. A T35 el hígado se caracterizó por exhibir hepatocitos claros, posiblemente producto del escaso contenido citoplasmático (Fig. 2). Además, el núcleo no mostró una coloración uniforme, sino que exhibió en su interior un material nuclear agregado, y un nucléolo de apariencia difusa. Se evidenciaron núcleos picnóticos y en algunos casos lucían fragmentados, lo que puede acarrear muerte celular por apoptosis. En general, los hepatocitos presentaron una citoarquitectura dañada, posiblemente producto de la temperatura de $35^{\circ} \mathrm{C}$ a la cual los peces fueron expuestos.

Histología de riñón: El riñón de T29 reveló un tejido normal semejante a lo descrito para otros teleósteos; se evidenció la presencia de glomérulos conteniendo eritrocitos en buen estado y con su respectiva cápsula de Bowman. Entre el glomérulo y la capsula de Bowman se observó el espacio de Bowman bien definido (Fig. 3). También se observaron células melanomacrófagas aisladas y el tejido hematopoyético $(\mathrm{TH})$ compacto y en buen estado.

En el riñón del grupo T18 se observó un glomérulo donde el complejo glomérulo venoso- espacio y capsula de Bowman, se presenta interrumpido, un posible edema en las venas que conforman el glomérulo (Fig. 3). Además, la capsula de Bowman se apreció muy

Fig. 2. Liver Cross section of C. macropomum exposed to different temperatures. $29^{\circ} \mathrm{C}$. Hepatocytesare observed $(\mathrm{H})$ with well-defined nuclei (dotted arrow) containing nucleoli (arrows). $\mathbf{1 8}^{\circ} \mathrm{C}$. It was observed clear hepatocytes (HC) and dark hepatocytes (HO). $\mathbf{3 5}^{\circ} \mathbf{C}$. Hepatocytes were observed with clear cytoplasm possibly as a result of loss of integrity of the cytoplasm. The nuclei does not show a uniform appearance (solid arrows), it noted diffuse nucleolus (dashed arrows) and the presence of picnotic nuclei (short arrows). 

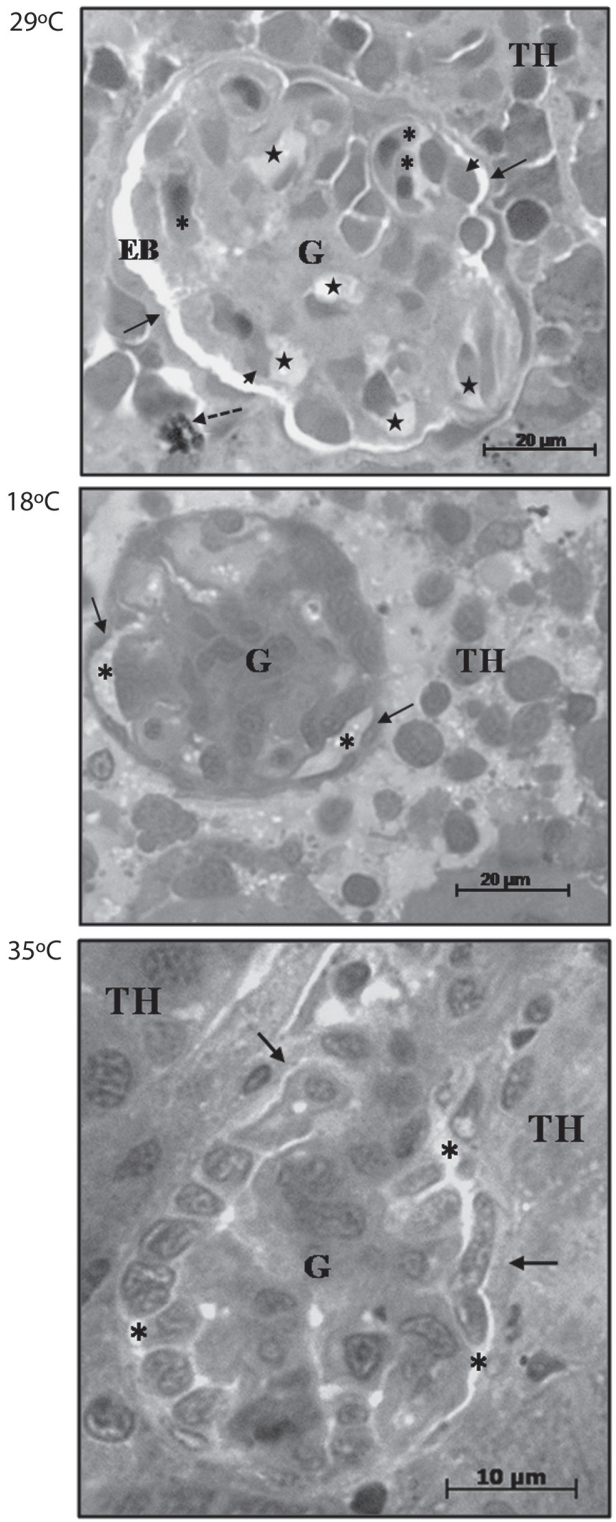

Fig. 3. Corte transversal de riñón de C. macropomum. $\mathbf{2 9}^{\circ} \mathrm{C}$. Detalle mostrando un glomérulo normal $(\mathrm{G})$ y cápsula de Bowman (flechas) rodeado de tejido hematopoyético homogéneo (TH). En el glomérulo se observan capilares (estrellas) con endotelio capilar en aparente buen estado (flechas cortas) y eritrocitos con morfología normal (asterisco). $\mathbf{1 8}^{\circ} \mathrm{C}$. Se observa una interrupción entre el glomérulo $(\mathrm{G})$ y la capsula de Bowman (flecha) quedando muy poco espacio entre los dos (asterisco), además se nota pérdida de la integridad del tejido hematopoyético (TH). $\mathbf{3 5}^{\circ} \mathrm{C}$. Se observaron glomérulos $(\mathrm{G})$ con el espacio de Bowman un poco reducido y el tejido hematopoyético homogéneo $(\mathrm{TH})$. delgada y se notó pérdida de la integridad del TH que la rodea evidenciada por su apariencia clara y alteraciones en la conformación y distribución celular.

En T35 se observó un glomérulo en aparente buen estado aunque el espacio de Bowman se aprecia muy reducido (Fig.3). El TH también parece no haberse afectado por la acción de una temperatura de $35^{\circ} \mathrm{C}$.

\section{DISCUSIÓN}

La distribución celular observada en las branquias de C. macropomun a T29 fue similar a la descrita para otras especies sugiriendo que esta temperatura se encuentra en el intervalo óptimo para el desarrollo de la especie la cual oscila entre 25 y $29^{\circ} \mathrm{C}$ (Torres et al. 2002). No así T18 en donde se observó alteración de los filamentos branquiales por la acumulación de lípidos, sugiriendo que se pudo afectar la función respiratoria de las branquias y con ello su propiedad osmorreguladora. Una de las reacciones generales de los poiquilotermos a la disminución de la temperatura es el aumento en los ácidos grasos insaturados y la acumulación de lípidos especialmente en las branquias tal y como ha sido demostrado en Sparusa urata expuesta a muy bajas temperaturas (Ibarz et al. 2005). Este cambio histológico-fisiológico inducido por la temperatura favorece la proliferación de algunos patógenos oportunistas (Aydin et al. 2009). Sin embargo, fue T35 la que indujo mayores alteraciones en la histología de

Fig. 3. Kidney cross section of C. macropomum exposed to different temperatures. $\mathbf{2 9}^{\circ} \mathrm{C}$. Detail showing anormal glomerulus $(\mathrm{G})$ and Bowman's capsule (arrows) surrounded by homogeneous hematopoietic tissue (TH). Glomerular capillaries are observed (stars) with endotheliumin apparent normal condition (short arrows) and erythrocytes with normal morphology (asterisk). $\mathbf{1 8}^{\circ} \mathrm{C}$. There is a discontinuity in the Bowman's space (asterisk) between the glomerulus (G) and Bowman's capsule (arrow), as well as noticeable loss of hematopoietic tissue integrity (TH). $\mathbf{3 5}^{\circ} \mathbf{C}$. It was observed glomeruli $(\mathrm{G})$ with reduced Bowman's space and homogeneous hematopoietic tissue (TH). 
los órganos evaluados; estos cambios anatomopatológicos, probablemente, provocaron alteraciones en los procesos respiratorios y disfuncionalidad en el papel de protección contra patógenos, desempeñado por las branquias del pez; esto, debido a que tanto los cilios como el mucus intervienen en la expulsión de microorganismos y sustancias irritantes que podrían ser peligrosos para la integridad del pez.

Walsh \& Ribelin (1975), formulan que el estado de separación del epitelio de la membrana basal de los filamentos branquiales es un artefacto introducido en el tejido poco antes o después de muerto el organismo, y no como resultado de los tratamientos químicos. Contrario a lo encontrado por estos autores, nuestros resultados muestran que los peces del grupo T29 no presentaron este fenómeno de pérdida o separación del epitelio branquial. Por lo que los cambios observados en el tejido branquial en T18 y T35 son el resultado de la exposición a esas temperaturas.

La citoarquitectura observada en el hígado T29 se corresponde con la reportada en la literatura para otras especies, tales como Salmo gairdneri (Stoker et al. 1985) y Serranus cabrilla (González et al. 1993). Roberts (1989) sostiene que los hepatocitos de los peces tienen una menor tendencia a disponerse en cordones, estando organizados en hileras de dos células como en la mayoría de los vertebrados inferiores.

La diferencia en la coloración de los hepatocitos de T18, probablemente se debió a la presencia de gran cantidad de vacuolas lipídicas en el interior de algunos de ellos, motivo por el cual el contraste de la coloración del tejido se modificó, tornándose más oscuros aquellos hepatocitos que presentaban mayor cantidad de vacuolas lipídicas en su interior y más claros aquellos que presentaban menor cantidad de lípidos. Esta condición podría deberse a una movilización de lípidos perivisceral hacia el hígado, lo que podría afectar la integridad de este tejido; tal y como ha sido demostrado por Ibarz et al. (2005) en el pez Sparusa urata, quienes reportaron que el contenido de lípidos aumentaba en estos organismos, al estar expuestos, por un período prolongado al frío. Estos autores observaron que ocurría una deposición de lípidos en el hígado, particularmente ácidos grasos 22:6n3y relacionaron los cambios en la morfología del tejido, tales como la presencia de hígados largos y amarillentos, con la deposición de estas moléculas.

La histología presentada en el hígado del grupo T18 sugiere que esta temperatura es crítica para la especie y de prolongarse podría conducir al desarrollo de daño oxidativo. En el hígado de la dorada Sparusa urata expuesta a bajas temperaturas, se ha reportado un incremento de $50 \%$ en la formación de especies reactivas del ácido barbitúrico lo que indica peroxidación lipídica, además un incremento en la producción de óxido nítrico y de proteínas tales como el factor de elongación alfa 1, de las proteínas inhibidora de la Raf-kinasa y de la tubulina los cuales son indicadoras de estrés oxidativo y de la inducción de apoptosis (Ibarz et al. 2010). También en especies de anguilas proveniente del mar del norte (Zoarces viviparus) y de la región polar (Pachycara brachycephalum) se observó que los parámetros indicadores de daño oxidativo (proteínas carboniladas, y productos reactivos del ácido barbitúrico (TBARS) se encontraron más elevados durante muy bajas temperaturas (Heise et al. 2007). Estos datos sustentan nuestras observaciones y demuestran que el hígado de los peces es sensible a daño oxidativo inducido por temperaturas por debajo de los rangos fisiológicos tolerados por cada especie.

Por otro lado, la T35 se puede considerar como una temperatura severa para el hígado de C. macropomum, donde el daño observado en el tejido podría ser el resultado de procesos apoptóticos y necróticos, sobrepasando la maquinaria antioxidativa de las células. Un incremento de TBARS y un altamente oxidado potencial redox junto con la reducción de la actividad de la enzima superoxido dismutasa fue observado en la anguila $Z$. viviparus expuesta a la temperatura severa de $26^{\circ} \mathrm{C}$ (Heise et al. 2006).

El riñón de C. macropomun expuesto a T29 reveló un tejido con morfología similar 
a la descrita en otros teleósteos (Charmi et al. 2009). Se observaron glomérulos bien constituidos y se observa la luz de los capilares intraglomerulares conteniendo eritrocitos de forma regular. Además, el espacio de Bowman se observó alrededor de todo el glomérulo.

A T18se observó una disminución del espacio de Bowman, probablemente producto de una alteración en el volumen del glomérulo venoso por edema endotelial. Los capilares intraglomerular es se encuentran revestidos por una serie de células endoteliales fenestradas extremadamente delgadas, con núcleos prominentes cuyo abultamiento se introduce en el lúmen del capilar (Zuasti et al. 1983, Lacy et al. 1987). Se infiere que una distención del endotelio capilar trajo como consecuencia una disminución en el tamaño de las fenestraciones alterando el papel de absorción de los glomérulos. Además, el edema endotelial conlleva a una disminución del diámetro del capilar trayendo como consecuencia isquemia y por consiguiente un problema de hipoxia que puede afectar el funcionamiento celular (Rojas et al. 2005).Por otra parte, se produjo pérdida de la integridad estructural del tejido hematopoyético ubicado en las zonas aledañas al glomérulo, de esta manera posiblemente se afectó la síntesis de hemoglobina en el pez, pudiendo inducir anemia. Esto se corresponde con los hallazgos reportados por Salazar-Lugo et al. (2009) quienes observaron una alteración en los parámetros hematológicos de $C$. macropomum, expuesta a $18^{\circ} \mathrm{Ccon}$ disminución de la hemoglobina y del hematocrito. El daño por temperatura también fue observado a T35 donde se evidenció una disminución del espacio de Bowman, probablemente producto de una alteración en el volumen del glomérulo venoso por edema endotelial.

En resumen, la temperatura de $18^{\circ} \mathrm{C}$ se puede considerar crítica para el pez C. macropomum y la de $35^{\circ} \mathrm{C}$ como severa. De los tres órganos evaluados, el hígado y las branquias resultaron ser los más sensibles a daños inducidos por estas temperaturas.

\section{AGRADECIMIENTOS}

Este trabajo fue financiado por el Fondo Nacional de Ciencias, Tecnología e Innovación proyecto UDO-FONACITG2005000775, agradecemos al Instituto de Investigaciones Biomédicas y Ciencias Aplicadas "Dra. Susan Tai" de la Universidad de Oriente, en especial a la Lic. Gilma Hernández por la realización de los cortes histológicos y a la piscicultora ALMA C.A. por suministrar los peces usados en el estudio.

\section{RESUMEN}

Colossoma macropomum es uno de los peces tropicales de agua dulce con mayor éxito en el cultivo en aguas continentales tropicales. Se realizó una evaluación histológica de branquias, hígado y riñón de este pez expuesto a tres temperaturas $\left(\mathrm{T} 18, \mathrm{~T} 29\right.$ y $\left.\mathrm{T} 35^{\circ} \mathrm{C}\right)$. Se utilizaron 18 ejemplares juveniles con índices biométricos de $17.87 \pm 7.88 \mathrm{~cm}$ y $87.69 \pm 34.23 \mathrm{~g}$. Los análisis histológicos fueron hechos de acuerdo a técnicas previamente descritas. Se encontró que las branquias, el hígado y el riñón de los peces T29 presentaron citoarquitectura normal. Por el contrario, en las branquias de los peces T18, se observaron gotas lipídicas inmersas en el tejido branquial; los peces T35 presentaron desorganización en la estructura del tejido branquial y necrosis celular. El hígado de los peces T18, mostró hepatocitos claros y oscuros y el de T35, presentó células con granulaciones citoplasmáticas y daño en la membrana plasmática. En el riñón de los T18, se observaron alteraciones en la distribución del tejido hematopoyético. La característica más resaltante en el riñón de los peces T35, fue la desorganización del tejido glomerular. En conclusión, la temperatura de $18^{\circ} \mathrm{C}$ se puede considerar crítica para la cachama y la de $35^{\circ} \mathrm{C}$ severa. De los tres órganos evaluados, el hígado y las branquias resultaron ser los más sensibles a los daños inducidos por las temperaturas en esta especie.

Palabras clave: Colossoma macropomum, histología, temperatura, hígado, riñón, branquias.

\section{REFERENCIAS}

Andrade de Pasquier, G., Y. Méndez \& D. Perdomo. 2011. Engorde experimental de cachama Colossoma macropomum) en la estación local el lago, estado Zulia, Venezuela. Zootecnia Trop. 29: 213-218.

Aydin, S., H. Gure, H. Cakici, S.Colakoglu \& R. Bircan. 2009. Gross pathology, blood chemistry, lipid and peroxide contents in rainbow trout (Oncorhynchus 
mykiss Walbaum) affected by experimental Arcobacter cryaerophilus infection at low water temperature. Acta Vet. Hung. 57: 305-17.

Azaza, M.S., M.N. Dhraief \& M.M. Kraiem. 2008. Effects of water temperature on growth and sex ratio of juvenile Nile tilapia Oreochromis niloticus (Linnaeus) reared in geothermal Waters in southern Tunisia. J. Thermal Biol. 33: 98-105.

Bravo, M.I., J. Medina, S. Marcano, H.J. Finol \& A. Boada-Sucre. 2005. Effects of herbicide on the kidneys of two Venezuelan cultured fish: Caquetaia kraussii and Colossoma macropomum (Pisces: Ciclidae and Characeae). Rev. Biol. Trop. 53: 55-60.

Cajaraville, M., M. Bebianno, J. Blasco, C. Porte, C. Sarasquete \& A. Viarengo. 2000. The use of biomarkers to assess the impact of pollution in coastal environments of the Iberian Peninsula: A practical approach. Sci. Total Environ. 47: 295-311.

Charmi, A., M. Bahmani, M.M. Sajjadi \& R. Kazemi. 2009. Morpho-histological study of kidney in farmed juvenile beluga, Husohuso (Linnaeus, 1758). Pakistan J. Biol. Sci. 12: 11-18.

Fernández, J. 1986. Peces más comunes del Territorio Federal Amazonas. Venezuela. Fon. Divul. 21: 29-35.

Goessling, W. \& T.E. North. 2011. Hematopoietic stem cell development: using the zebrafish to identify the signaling networks and physical forces regulating hematopoiesis. Methods in Cell Biology. Vol. 105. Cap. 5. 117-136.

González, G., S. Crespo \& J. Brusle. 1993. Histocitological study of the live of the cabrilla sea bass, Serranus cabrilla (Teleostei, Serranidae), an avaible model for marine fish experimental studies. J. Fish. Biol. 43: 363-373.

Heise, K., S. Puntarulo, M. Nikinmaa, D. Abele \& H.O. Pörtner. 2006. Oxidative stress during stressful heat exposure and recovery in the north sea eelpout Zoarces viviparus. J. Exp. Biol. 209: 353-63.

Heise, K., M.S. Estevez, S. Puntarulo, M. Galleano, M. Nikinmaa, H.O. Pörtner \& D. Abele. 2007. Effects of seasonal and latitudinal cold on oxidative stress parameters and activation of hypoxia inducible factor (hif-1) in zoarcid fish. J. Comp. Physiol. B. 177: 765-77.

Ibarz, A., J. Blasco, M. Beltrán, M.A. Gallardo, J. Sánchez, R. Salab \& J. Fernández-Borras. 2005. Cold-induced alterations on proximate composition and fatty acid profiles of several tissues in gilthead sea bream (Sparusa urata). Aquaculture 249: 477-486.

Ibarz, A., M. Martín-Pérez, J. Blasco, D. Bellido, E. De Oliveira \& J. Fernández-Borrás. 2010. Gilthead sea bream liver proteome altered at low temperatures by oxidative stress. Proteomics 10: 963-75.
Körner, O., S. Kohno, R. Schönenberger, M.J. Suter, K. Knauer, L.J. Jr. Guillette \& P. Burkhardt-Holm. 2008. Water temperature and concomitant waterborne ethinylestradiol exposure affects the vitellogenin expression in juvenile brown trout (Salmo gairdneri). Aquat. Toxicol. 90: 188-96.

Lacy, E.R.M. Castelucci \& E. Reale. 1987. The elasmobranch renal corpuscle: fine structure of Bowman's capsule and the glomerular capillary wall. Anat. Rec. 218: 294-305.

Luft, J.H. 1961. Improvements in epoxy resin embedding methods. J. Biophys. Biochem. Cytol. 9: 409.

Marcogliese, D.J. 2008. The impact of climate change on the parasites and infectious diseases of aquatic animals. Rev. Sci. Tech. 27: 467-84.

Perez-Casanova, C., M.L. Rise, B. Dixon, L.O.B. Afonso, J.R. Hall, S.C. Johnson \& A.K. Gamper. 2008. The immune and stress responses of Atlantic cod to long-term increases in water temperature. J. Fish \& Shellfish Immunol. 24: 600-60.

Roberts, R. 1989. The pathophysiology and systematic pathology of teleosts. Fish Pathology, p. 56-134. R.J. Roberts (ed.). Bailliere Tindall, London, England.

Rojas, L.M., R. Mcneil, T. Cabana \& P. Lachapelle. 1997. Diurnal and nocturnal visual function in two tactile foraging waterbirds. The American white ibis and the black skimmer. Condor 99: 191-200.

Rojas, L.M., K. Benítez, S.M. Suárez, A. Boada-Sucre, Y. Ramírez, M.A. Romero, G. Hernández. 2005. Ultraestructura de la retinopatía causada por la hiperoxia en ratas en desarrollo. Rev. Invest. Clin. 57: 794-80.

Rougeot, C., C. Prignon, C.V. Ngouana \& C. Mélard. 2008. Effect of high temperature during embryogenesis on the sex differentiation process in the Nile tilapia, Oreochromis niloticus, Aquaculture 276: 205-208.

Salazar-Lugo, R., A. Estrella, A. Oliveros, E. RojasVillarroel, L. Villalobos De B \& M. Lemus. 2009. Paraquat and temperature affect nonspecific immune response of Colossoma macropomum, Environ. Pharmacol. Toxicol. 27: 321-326.

Sano, M., T. Ito, T. Matsuyama, C. Nakayasu \& J. Kurita. 2009. Effect of water temperature shifting on mortality of Japanese flounder Paralichthys olivaceus experimentally infected with viral hemorrhagic septicemia virus. Aquaculture 286: 254-258.

Stoker, P., J. Larsen, G. Booth \& M. Lee. 1985. Pathology of gill and liver tissues from two genera of fishes exposed to two coal-derived materials. J. Fish Biol. 27: 31-46.

Tafur-Gonazález, J., F. Alcántara, M. Pizarro, R. Cubas, L. Mori-Pinedo \& F. Chu-Koo. 2009. Ipaco Piaractus brachypomus y gamitana Colossoma macropomum criados en policultivo con el bujurqui-tucunaré, 
Chaetobranchus semifasciatus (Cichlidae). Instituto de investigaciones de la Amazonía peruana vol. 18 $n^{\circ}$ 1-2: 97-104.

Torres, J., O. Castillo, G. Cortez, J. Bravo \& M. Fontaine. 2002. Prevalencia de trematodos monogésicos branquiales en cachamas Colossoma macropomum en la Estación Piscícola Papelón. VI Congreso Venezolano de Acuicultura. San Cristóbal, Venezuela.

Useche, M. 2000. El cultivo de la cachama, manejo y producción. Primer taller piscícola 2000. Universidad Experimental del Táchira. Decanato de
Investigaciones San Cristóbal. Venezuela. (Consultado: 27 agosto 2012, www.unet.edu.ve/ frey/varios/ dinv/piscicultura/cachama/).

Walsh, A. \& W. Ribelin. 1975. The pathology of the pesticide poisoning, p 497-517. In W.E. Ribelin \& G. Migaki (eds.). The pathology of fishes. University of Wisconsin, Wisconsin, EE.UU.

Zuasti, A., B. Agulleiro \& F. Hernandez. 1983. Ultraestructure of the kidney of the marine teleost Sparusa uratus; the renal corpuscle and the tubular nephron. Cell Tissues Res. 228: 99-106. 